\title{
Nivel de actividad física, masa y fuerza muscular de mujeres mayores dela comunidad: Diferencias por grupo etario \\ Physical activity level, muscle mass and strength of community elderly women: Differences by age group María Cristina Enríquez-Reyna, Daniel Carranza Bautista, Ricardo Navarro Orocio \\ Universidad Autónoma de Nuevo León (México)
}

Resumen. Se propuso describir las diferencias por grupo etario del nivel de actividad física, la cantidad de masa y fuerza muscular de mujeres mayores de la comunidad. Esto fue un estudio descriptivo-comparativo de 415 mujeres mayores que acuden a un club público de adultos mayores del área urbana. Se aplicaron el Cuestionario de Actividad Física para Adultos Mayores, análisis de bioimpedancia eléctrica y la Batería Corta de Desempeño Físico. Se presentan descripciones con promedios y percentiles por grupo etario. Se estimó que más de la mitad de las participantes reportó un nivel de actividad física bajo (62\%). Una fuerza de prensión manual baja en 37.5\% y 43.6\% velocidad de marcha de riesgo (menor a 1 m/s). En esta muestra, el nivel de actividad física mostró variabilidad en su asociación con la masa muscular, y la fuerza de miembros superiores e inferiores. Con el avance de la edad, la masa y fuerza musculares disminuyeron paulatinamente $(p<.01)$.

Palabras clave. Ejercicio, salud de la mujer, fuerza de la mano, fuerza muscular, México.

Abstract. The aim was to describe age-group related differences in physical activity levels, the amount of muscle mass and strength in elderly. of 415 elderly women from an urban public seniors' club participated in this study with a descriptive-comparative character. The Physical Activity Scale for the Elderly, an electrical bioimpedance analysis, and the Short Physical Performance Battery were applied. Descriptions are presented as means and percentiles by age group. As the results demonstrate, more than half of the participants reported a low level of physical activity (62\%). Furthermore, a low manual grip strength $37.5 \%$ and $43.6 \%$ a gait speed risk (less than $1 \mathrm{~m} / \mathrm{s}$ ) were found respectively. In this sample, the level of physical activity showed variability in its association with muscle mass, and upper and lower limb strength. With advancing age, muscle mass and strength gradually decreased $(p<.01)$.

Keyword. Exercise, women's health, hand strength, muscle strength, Mexico.

\section{Introducción}

Con las alteraciones del ambiente hormonal durante la vejez, se contribuye a la aparición del fenotipo sarcopénico que desencadena disminución de la capacidad física y a la larga, pérdida de la independencia funcional (Carrillo-Esper, et al., 2011; Mata-Ordóñez, et al., 2013). La sarcopenia, constituye la pérdida de masa muscular y disminución de la función muscular relacionada con el envejecimiento (Cruz-Jentoft, et al., 2010; Patil, et al., 2013). Debido a que la masa y la fuerza muscular muestran una relación no lineal, para el diagnóstico de sarcopenia se sugiere la medición de ambos criterios (Cruz-Jentoft, et al., 2010; Goodpaster, et al., 2006). Sin embargo, mayor cantidad de masa muscular no implica un mejor rendimiento físico o mejor calidad muscular, se ha reportado que la calidad de la masa muscular es influida por otros factores como la capacidad cardiopulmonar, el padecer enfermedades ortopédicas (Chien, Kuo, \& Wu, 2010), la capacidad física (Li-Kuo, et al., 2013), la raza (Zhao, Wang, Zang, Hua, He, \& Zhu, 2013), edad y sexo (Hayashida, Tanimoto, Takahashi, Kusabiraki, \& Tamaki, 2014; Zhao, et al., 2013).

Después de los 50 años, la cantidad de masa muscular disminuye del uno a dos por ciento por año (Abellan, 2009); y para los 70's, el declive de fuerza muscular acumulado pudiera ser del 20 al 40\% (Berger \& Doherty, 2010). En vista de la feminización del envejecimiento, la fuerte influencia de factores biológicos y que la dependencia se presenta con mayor frecuencia en mujeres, la situación de las adultas mayores en México resulta preocupante. Las encuestas poblacionales hacen patente su vulnerabilidad, en este subgrupo poblacional considerado físicamente inactivo (López, et al., 2016), se presenta mayor incidencia de obesidad abdominal, anemia y enfermedades crónicas (Instituto Nacional de Salud Pública [INSP], 2012; Secretaría de Salud/INSP, 2016) con afectación directa sobre la movilidad física (Alva, Camacho, Velázquez, \& Lazarevich, 2013). El factor cultural también afecta, ya que las adultas mayores han considerado la pérdida de la independencia como propia del envejecimiento (González-Célis, 2010).

El incremento de las dificultades para mantener la autonomía individual de las mujeres mayores puede afectar la estabilidad social a múltiples niveles. Surge la inquietud de realizar este proyecto debido a

Fecha recepción: 11-09-17. Fecha de aceptación: 05-06-18

María Cristina Enríquez-Reyna

maria.enriquezryn@uanl.edu.mx que las encuestas nacionales se han limitado a describir el estatus nutricional y sus consecuencias en la funcionalidad física estimadas a través de cuestionarios o medidas indirectas de la capacidad física. Solo es posible localizar algunos reportes con datos sobre la capacidad física de población adulta mayor a partir de muestras pequeñas con alguna característica o patología específica como la sarcopenia. El análisis de la relación entre el nivel de actividad física, la cantidad y calidad de la masa muscular permitirá la detección oportuna de datos de deterioro en mujeres mayores de la comunidad. Además de orientar la planeación y ejecución de estrategias para la adecuada prescripción del ejercicio en este grupo poblacional. Se plantearon dos objetivos específicos: 1 . Describir las características personales, el nivel de actividad física, masa y fuerza muscular de adultas mayores de la comunidad por grupo etario; y 2. Estimar la relación entre el nivel de actividad física y variables relativas a la masa y fuerza musculares.

\section{Material y método}

\section{Diseño del estudio}

Estudio cuantitativo no experimental con diseño descriptivo-comparativo de corte transversal.

\section{Participantes}

El universo de estudio estuvo conformado por 2701 adultos mayores que acuden a cinco Casas Club del Programa Adulto Mayor del Sistema para el Desarrollo Integral de la Familia (DIF). El tamaño de muestra fue calculado a través del paquete nQuery Advisor 4.0; para una prueba de correlación bilateral con parámetro conservador pequeño (0.20), nivel de confianza del 95\%, límite de error estimado de .05 y potencia del 90\%. Se utilizó un muestreo aleatorio simple; durante la recolección, se invitó diariamente a la misma proporción de participantes por lo que todas las asistentes de la casa club tuvieron la misma probabilidad de ser seleccionadas para participar. Se evaluaron 415 participantes que constituyen el total de la muestra.

Se incluyó a mujeres de 60 a 85 años, con integridad cognitiva de acuerdo al Cuestionario de Pfeiffer (Pfeiffer, 1975), con independencia en Actividades Básicas de la Vida Diaria de acuerdo al índice de Barthel (Mahoney \& Barthel, 1965), que refirieran capacidad de caminar distancias cortas sin ayuda de otra persona, sin contraindicación expresa para el ejercicio y que aceptaran participar voluntariamente. Se excluyó a personas que utilizan marcapasos debido a que no se les puede realizar el análisis de bioimpedancia. Se eliminaron los datos de participantes 
que no lograron terminar la prueba de la silla en cinco ocasiones. Las candidatas que presentaron algún grado de deterioro cognitivo o dependencia en actividades de la vida diaria, no participaron en el estudio; esto se evaluó con el propósito de incluir de forma exclusiva a mujeres mayores que puedan desenvolverse con autonomía en la comunidad.

\section{Cuestionarios}

Se diseñó una cédula de datos personales para cuestionar aspectos asociados al nivel de actividad física en personas mayores (Lera-López, Garrues, \& Suárez, 2017). La cédula incluyó siete preguntas cortas acerca de la edad, escolaridad, estado civil (con o sin pareja), número de convivientes habituales, caídas en el último año, número de padecimientos reconocidos y de medicamentos de uso habitual. Además, con la versión en español de la Physical Activity Scale for the Elderly (Washburn, Smith, Jette, \& Janney, 1993) se estimó la cantidad de actividad física en METs (unidades de gasto energético). La escala cuestiona sobre la actividad física realizada en el hogar, con fines recreativos y de ejercicio. A partir de la consideración de la posición en que se realiza la actividad, y la frecuencia de realización, se estima el gasto energético que estas actividades representan para el sujeto. Esta escala ha sido ampliamente utilizada en investigación con adultos mayores debido a su sensibilidad para distinguir niveles bajos de actividad física.

\section{Mediciones}

Para medir la masa muscular, se realizó análisis de bioimpedancia eléctrica con el analizador TANITABC-418 (Inner Scan TM, Arlington Heights, IL, USA) identificado por usar ecuaciones validadas con métodos de referencia estándar (Dehghan \& Merchant, 2008). Se solicitó que las participantes tuvieran al menos dos horas de ayuno, que no hubieran consumido bebidas alcohólicas o realizado ejercicio durante las últimas ocho horas y que realizaran vaciamiento de vejiga antes de la medición. Primero se midió la talla en centímetros con estadímetro SECA, luego se introdujeron los datos al analizador y se pesó a la participante. Se consideró el valor escalar que reporta al respecto del peso (kg), índice de masa corporal, masa musculo-esquelética apendicular (sumatoria de masa muscular de extremidades) y posteriormente se estimó un índice (masa muscular apendicular esquelética/talla²).

\section{Pruebas físicas}

Se distinguió entre fuerza de miembros superiores (fuerza manual máxima) e inferiores (velocidad de marcha y rendimiento físico a partir de la Batería Corta de Desempeño Físico, SPPB por sus siglas en inglés). La fuerza de prensión manual máxima es un marcador isométrico de fuerza medida con dinamómetro; en mujeres se considera que $20 \mathrm{~kg}$ de fuerza indica normalidad(Lauretani, et al., 2003). Para la medición, se ajustó el dinamómetro a la mano de la participante; se realizó un ensayo y prueba definitiva por cada mano; de los cuatro datos, se eligió el valor más alto como indicador de la fuerza de prensión manual para los análisis.

Después se aplicó la SPPB que incluye la evaluación de la prueba de la silla, equilibrio y velocidad de marcha con cronómetro digital Casio HS70W. Se determina el desempeño en cada prueba con una puntuación de 0 a 4 puntos, se suman los resultados parciales y se considera entonces una puntuación de 0 a 12 puntos; a mayor puntuación mejor rendimiento físico; el criterio de evaluación sugerido señala que de cero a seis puntos el rendimiento físico es bajo, de siete a nueve de nivel intermedio y de 10 o más, nivel alto (Guralnik, et al., 1994). En la prueba de la silla se cuantifica el tiempo en segundos que se tarda en levantarse de una silla y volver a sentarse en cinco ocasiones; para la puntuación se consideran los parámetros que señalan los autores de la batería. Se considera que, un menor tiempo de realización en esta prueba, es debido a que se tiene mayor fuerza. En las participantes que mostraban algún grado de fragilidad se omitió la medición debido a la dificultad de la prueba; sus datos se eliminaron de los análisis por lo que no formaron parte de la muestra final de este estudio.

La prueba de equilibrio examina la capacidad para mantenerse de pie con los pies juntos al lado de otro, en semitándem y tándem comple- to (diez segundos por posición). Quienes son capaces de mantener la posición tándem completo durante diez o más segundos obtienen cuatro puntos en esta prueba.

Para la velocidad de marcha se midió el tiempo transcurrido desde la señal de inicio de la medición hasta el momento en que el participante cruzó la línea marcada para los cuatro metros que se evaluaron. Se solicitó al participante situarse de pie, con los pies paralelos delante de la línea de salida, se le indicó que caminara de manera habitual para dar dos vueltas a un circuito de seis metros delineado con conos de tránsito. Para no considerar la velocidad de arranque o de frenado, se cronometró exclusivamente el tiempo de traslado entre los 4 metros centrales del recorrido. En esta prueba también se considera que a menor tiempo de traslado mayor fuerza, se considera pertinente una velocidad de marcha habitual de $0.8 \mathrm{~m} / \mathrm{s}$ (Lauretani, et al., 2003).

\section{Procedimiento}

Para la realización de este estudio, se obtuvo la aprobación del Comité de Bioética en Investigación en Ciencias de la Salud de la Facultad de Organización Deportiva, Universidad Autónoma de Nuevo León, México. Asimismo, para la recolección de datos, se obtuvo el permiso de las autoridades de los centros estatales para el Desarrollo Integral de la Familia. Se acudió a la Casa Club donde se realizó una reunión con el personal y posteriormente con la población de adultos mayores. Cuando se informó a las posibles participantes del estudio, se procedió a revisar el cumplimiento de los criterios de selección. Alas candidatas que no cumplían con los criterios de inclusión, se les ofreció la medición del análisis corporal y/o las pruebas físicas como agradecimiento por su interés de participar en el estudio. Se firmó el consentimiento informado, se llenó la cédula de datos personales, mediciones y pruebas físicas. A todas las candidatas y participantes se les entregaron resultados de sus pruebas y un tríptico informativo como retribución por su voluntad de participar.

\section{Análisis estadístico}

Los resultados se analizaron con el paquete estadístico SPSS versión 21.0 (Statistics, 2012). Se dividió la muestra por grupos de edad para describir los cambios en la cantidad y calidad muscular en percentiles. Se utilizó estadística descriptiva para describir las características de las participantes, nivel de actividad física, masa y fuerza muscular (media, mediana, desviación estándar, valormínimo y máximo). Se determinó la distribución de los datos de las variables cuantitativas mediante la prueba de bondad de ajusteKolmogorov-Smirnov con corrección deLilliefors. Se realizó análisis de factor por grupo etario para estimar la significancia de la variabilidad entre las variables descritas. Finalmente se corrieron pruebas de correlación para estimar la relación entre el nivel de actividad física y el resto de las variables de estudio.

\section{Resultados}

Se presentan las características personales y nivel de actividad física por grupo etario (Tabla 1).

\begin{tabular}{|c|c|c|c|c|c|c|}
\hline Variable/Grupo & $\begin{array}{l}60-64 \\
n=86 \\
n\end{array}$ & $\begin{array}{c}65-69 \\
n=113\end{array}$ & $\begin{array}{c}70-74 \\
n=111\end{array}$ & $\begin{array}{l}75-79 \\
n=72 \\
\end{array}$ & $\begin{array}{c}=80 \\
n=33\end{array}$ & $\begin{array}{l} \\
p\end{array}$ \\
\hline Edad, años & $62.43 \pm 1.3$ & $66.96 \pm 1.4$ & $71.88 \pm 1.3$ & $76.75 \pm 1.4$ & $82.76 \pm 2.8$ & ** \\
\hline Escolaridad, años & $7.07 \pm 3.4$ & $5.78 \pm 3.2$ & $4.77 \pm 3.8$ & $4.82 \pm 3.3$ & $3.64 \pm 3.1$ & ** \\
\hline Convivientes, num & $2.38 \pm 1.9$ & $2.25 \pm 1.9$ & $2.04 \pm 1.8$ & $2.32 \pm 1.8$ & $2.21 \pm 1.6$ & \\
\hline Caídas, num & $0.70 \pm 1.2$ & $1.01 \pm 1.7$ & $1.03 \pm 1.8$ & $1.01 \pm 1.9$ & $0.94 \pm 2.0$ & \\
\hline Padecimientos, num & $1.58 \pm 1.1$ & $1.93 \pm 1.4$ & $1.72 \pm 1.2$ & $2.04 \pm 1.7$ & $1.64 \pm 1.1$ & \\
\hline Medicamentos, num & $2.24 \pm 1.5$ & $2.56 \pm 1.7$ & $2.42 \pm 1.7$ & $2.31 \pm 1.8$ & $2.12 \pm 1.3$ & \\
\hline Peso, $k g$ & $71.57 \pm 13.8$ & $67.48 \pm 14.5$ & $66.33 \pm 11.2$ & $62.77 \pm 10.2$ & $60.06 \pm 9.1$ & ** \\
\hline Talla, $\mathrm{cm}$ & $1.52 \pm 6.3$ & $1.50 \pm 5.9$ & $1.50 \pm 6.4$ & $1.49 \pm 6.5$ & $1.47 \pm 5.0$ & ** \\
\hline IMC, $\mathrm{kg} / \mathrm{m}^{2}$ & $30.98 \pm 5.5$ & $30.40 \pm 5.3$ & $29.59 \pm 5.0$ & $28.20 \pm 4.3$ & $27.62 \pm 3.8$ & ** \\
\hline $\begin{array}{l}\text { Nivel de actividad } \\
\text { física, } M E T s\end{array}$ & $8.59 \pm 4.6$ & $9.09 \pm 5.7$ & $8.19 \pm 4.4$ & $7.72 \pm 3.9$ & $8.14 \pm 3.7$ & \\
\hline
\end{tabular}

En relación a otras características descriptivas de la muestra, el 61.2\% de las participantes señaló no tener pareja; sólo el 15.9\% comentó que vivía sola. Un $11.8 \%$ reportó no haber cursado educación formal, 
sin embargo, la mayoría de ellas confirmó saber contar y firmar. El $12.5 \%$ refirió no sufrir ningún padecimiento, el $11 \%$ señaló no consumir medicamentos. El 40\% mencionó haber sufrido alguna caída durante el último año.

El percentil .25 de la muestra completa se puntuó 4.97 METs de actividad física habitual; y el percentil .75, acumuló 11.11 METs. Los autores del cuestionario señalan punto de corte de 9.5 para identificar a personas sedentarias, de tal suerte que el $69.6 \%$ de las participantes se clasifican con valores inferiores a ese corte. En la Tabla 2 se describen los cambios en la cantidad y calidad muscular en percentiles por grupo de edad.

\begin{tabular}{|c|c|c|c|c|c|c|}
\hline Grupo & & & $\begin{array}{l}\text { IMMAE, } \\
\mathrm{kg} / \mathrm{m}^{2}\end{array}$ & $\begin{array}{c}\text { Fuerza } \\
\text { manual } \\
\text { máxima, } k g\end{array}$ & $\begin{array}{l}\text { Prueba de la } \\
\text { silla, } s\end{array}$ & $\begin{array}{l}\text { Velocidad de } \\
\text { marcha, s }\end{array}$ \\
\hline \multirow{5}{*}{$\begin{array}{l}60-64 \text { años } \\
n=86\end{array}$} & \multicolumn{2}{|c|}{ Media } & 7.13 & 20.59 & 11.69 & 4.58 \\
\hline & & .25 & 6.72 & 18.10 & 9.15 & 3.37 \\
\hline & \multirow[t]{2}{*}{ Percentil } & .50 & 7.14 & 21.15 & 11.17 & 4.18 \\
\hline & & .75 & 7.63 & 23.35 & 12.90 & 5.38 \\
\hline & \multicolumn{2}{|c|}{ Media } & 7.48 & 23.24 & 10.89 & 3.81 \\
\hline 65-69 años & & .25 & 6.99 & 18.93 & 8.72 & 3.20 \\
\hline \multirow{3}{*}{$n=113$} & \multirow[t]{2}{*}{ Percentil } & .50 & 7.52 & 23.05 & 10.35 & 3.57 \\
\hline & & .75 & 8.02 & 27.43 & 11.84 & 4.03 \\
\hline & \multicolumn{2}{|c|}{ Media } & 7.55 & 22.24 & 10.69 & 3.95 \\
\hline \multirow{4}{*}{$\begin{array}{l}\text { 70-74 años } \\
n=111\end{array}$} & & .25 & 6.96 & 19.30 & 9.04 & 3.40 \\
\hline & \multirow[t]{2}{*}{ Percentil } & .50 & 7.48 & 22.20 & 10.31 & 3.85 \\
\hline & & .75 & 8.03 & 24.90 & 12.39 & 4.50 \\
\hline & \multicolumn{2}{|c|}{ Media } & 7.38 & 21.60 & 11.27 & 4.22 \\
\hline 75-79 años & & .25 & 6.80 & 18.90 & 9.00 & 3.20 \\
\hline \multirow{3}{*}{$n=72$} & Percentil & .50 & 7.27 & 21.60 & 11.00 & 3.96 \\
\hline & & .75 & 7.98 & 24.40 & 13.00 & 5.00 \\
\hline & \multicolumn{2}{|c|}{ Media } & 6.97 & 19.63 & 12.32 & 4.54 \\
\hline$=80$ años & & .25 & 6.53 & 16.40 & 10.21 & 3.72 \\
\hline \multirow[t]{2}{*}{$n=33$} & Percentil & .50 & 6.92 & 19.40 & 12.40 & 4.47 \\
\hline & & .75 & 7.41 & 23.25 & 14.89 & 5.22 \\
\hline $\begin{array}{l}\text { Diferencias } \\
\text { por grupo } \\
\text { etario }\end{array}$ & $p$ & & $* *$ & $* *$ & & $* *$ \\
\hline
\end{tabular}

En la muestra completa, con excepción del índice de masa musculoesquelética apendicular y la fuerza de prensión manual máxima, la distribución de los datos mostró valores de $p<.02$, por lo tanto, se rechaza la hipótesis nula de normalidad y se concluye que no muestran distribución normal. Se corrieron entonces pruebas de correlación de Spearman para explorar la significancia de las relaciones entre el nivel de actividad física y las otras variables de estudio. En la tabla 3 se presentan los resultados del análisis de correlación entre el nivel de actividad física y las principales variables de estudio por grupo etario.

Tabla 3.

Relación entre el nivel de actividad física y otras variables por grupo etario

\begin{tabular}{lccccc} 
Relación entre el nivel de actividad fisica y otras variables por grupo etario \\
\hline \multirow{2}{*}{ Nivel de actividad física, METs/ } & $60-64$ & $65-69$ & $70-74$ & $75-79$ & $=80$ \\
& $n=86$ & $n=113$ & $n=111$ & $n=72$ & $n=33$ \\
\hline IMMAE, $\mathrm{kg} / \mathrm{m} 2$ & -.103 & -.153 & $-.269^{* *}$ & -.137 & -.074 \\
Fuerza manual máxima, $\mathrm{kg}$ & .183 & $.378^{* *}$ & .085 & .100 & .009 \\
Prueba de la silla, $\mathrm{s}$ & -.018 & $-.213^{*}$ & $-.213^{*}$ & $-.368^{* *}$ & -.220 \\
Velocidad de marcha, $s$ & -.177 & -.124 & $-.280^{* *}$ & $-.326^{* *}$ & -.164 \\
Rendimiento físico, $\mathrm{pts}$ & .206 & .092 & $.285^{* *}$ & $.277^{*}$ & .182 \\
\hline Nent & .20
\end{tabular}

Rendimiento físico, $p t s$

Nota: $n=415$. METs=unidades de gasto energético; IMMAE = índice de masa musculo-

esquelética apendicular. ${ }^{*} p<.05,{ }^{, *} p<.01$.

El35.7\% de la muestra presentó fuerza manual máxima menor a 20 kg., lo que sugiere baja fuerza muscular. La velocidad de marcha habitual del $43.6 \%$ de las participantes fue menor a $1 \mathrm{~m} / \mathrm{s}$ lo que se ha asociado a riesgo de caídas. Y el rendimiento físico en base al corte de seis puntos

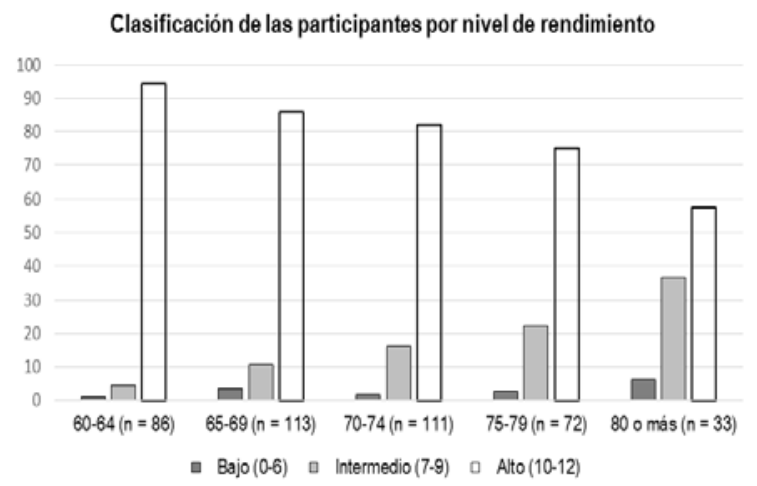

Figura 1. Clasificación de las participantes de acuerdo al nivel de rendimiento físico (Short Physical Performance Battery) por grupo etario. que sugieren los autores de la SPPB, fue bajo en una de cada diez participantes.

\section{Discusión}

Se presenta información a detalle acerca de la relación entre el nivel de actividad física, masa y fuerza muscular de 415 mujeres mayores independientes de la comunidad. Los análisis descriptivos se consideran representativos del noreste de México y exponen el declive por grupo etario en las variables del estudio.

La muestra presenta características concordantes con las participantes del último censo nacional al respecto de este grupo poblacional en lo relativo a la edad promedio de 70 años, escolaridad de primaria incompleta y tasa de analfabetismo baja (Instituto Nacional de Estadística y Geografía, 2010). En contraste, la proporción de participantes que viven sin pareja es superior al reporte de la Encuesta Nacional de Nutrición y Salud (INSP, 2012); esto puede explicarse con el reporte que señala que actualmente en México la esperanza de vida ha aumentado y es mayor para las mujeres (79.77 años), lo que suscita la reconocida feminización del envejecimiento. Se considera entonces que muchas de las participantes de esta muestra sobreviven a sus esposos. Debido a que vivir en estado de viudez se ha identificado como un estatus de vulnerabilidad, pérdida de la identidad, soledad y enfermedad (Montes de Oca, 2011), deben considerarse estos rasgos específicos de la muestra. La prevalencia de caídas en el último año (40\%) concuerda con otros reportes del contexto que señalan un promedio del 34 al 58\% (Enríquez-Reyna, et al., 2013; Leiva-Caro, et al., 2015).

El relativamente bajo nivel de actividad física en METs concuerda con otros reportes del país (INSP, 2012; Medina, Janssen, Campos, \& Barquera, 2013; Salvo, et al., 2015). No haber encontrado diferencias por grupo etario en el nivel de actividad física de las participantes, puede deberse a que, de manera independiente a la edad, la muestra proviene de un club público de adultos mayores cuyo criterio de aceptación es que los asistentes sean independientes para sus actividades básicas de la vida diaria. De manera específica, la tendencia a la inactividad física durante el envejecimiento puede explicarse por la cultura física preponderante a nivel nacional en esa etapa de la vida; se ha reportado que las personas de 60 a 69 años tienden a ser 1.58 veces más inactivas que las de 20 a 29 años (Medina, et al., 2013).

La inactividad física es considerada el cuarto lugar entre los factores de riesgo de mortalidad a nivel mundial (Kohl, et al., 2012); durante el envejecimiento se ha asociado con características sociodemográficas adversas, falta de interacción social, mala salud (Souza, et al., 2015), además de multimorbilidades (Dhalwani, et al., 2016; Ortlieb, et al., 2014). En contraste, el mantenimiento de los niveles de actividad física ha demostrado su utilidad para reforzar la dimensión social de los adultos mayores (Benedetti, Schwingel, \& Torres, 2011; Bohorquez, Lorenzo, \& García, 2014) misma que juega un papel determinante para la práctica de actividad física en mujeres mayores (Tuero \& GonzálezBoto, 2018). Dada la conexión entre la práctica de ejercicio físico, el potencial creativo (Cantarero \& Carranque, 2016) y otros beneficios psicológicos (Guillén \& Angulo, 2016), habría de promoverse la participación de la población adulta mayor en este tipo de actividades para establecer condiciones favorables a la condición física e incidir en un aumento de la autonomía durante la vejez.

Otros autores han explicado la disminución de la masa y fuerza muscular observable duranteel envejecimiento (Berger \& Doherty, 2010; Cruz-Jentoft, et al., 2010; Dodds, Kuh, Aihie, \& Cooper, 2013; Granic, et al., 2016). La relación inversamente proporcional entre la edad y variables asociadas a la masa y fuerza muscular ha sido reportada previamente en estudios realizados en España (Cabrero-García, et al., 2012; Leyton, Luis-del-Campo, Morenas, \& Roldán, 2012). De la misma forma que en otros contextos, se corroboró la asociación positiva del nivel de actividad física, la masa y fuerza muscular (Bann, et al., 2015; Chien, et al., 2010; Dodds, et al., 2013; Granic, et al., 2016; Klee, Alberici, Schûler, \& González, 2015). Así, es posible comprender los aspectos relativos a la cantidad y calidad muscular asociados en mujeres 
mayores de la comunidad.

Los hallazgos de este estudio no incluyen la descripción de hombres o personas institucionalizadas lo que representa una limitación de este trabajo. Otra limitación es la falta de uso de equipos especializados para la medición de la composición corporal y las capacidades físicas consideradas. Sin embargo, los equipos utilizados han mostrado su confiabilidad para muestras poblacionales y son accesibles para la práctica clínica; esto con el fin de promover la inclusión de la medición de estas variables en estudios epidemiológicos. Considerando la influencia determinante del contexto, la raza y la subjetividad de los cuestionarios, futuros análisis deberían considerar muestras poblacionales de mayor proporción, población de ambos sexos, el seguimiento longitudinal y/o experimental para un mejor conocimiento del comportamiento de estas variables en este creciente grupo poblacional. Una mejor comprensión de los mecanismos de deterioro y tratamiento de esta problemática servirá como referencia para el diseño de estrategias de intervención adecuadas a las necesidades de la población del contexto.

\section{Conclusiones}

Se describe la influencia de la edad sobre el nivel de actividad física, masa y fuerza muscular en mujeres mayores de la comunidad. En esta muestra, la cantidad de masa muscular mostró asociación con la fuerza de miembros superiores, inferiores y el rendimiento físico. Con el incremento de la edad, la masa y fuerza musculares disminuyeron paulatinamente. Esta publicación presenta información orientadora acerca de la normalidad/deterioro de las pruebas físicas aplicadas en mujeres ancianas del noreste de México.

\section{Referencias}

Abellan, V.K. G. (2009). Epidemiology and consequences of sarcopenia. Journal of Nutrition Health and Aging, 13(8), 708-12. Recuperado de: https://doi.org/10.1007/s12603-009-0201-z

Alva, M. V., Camacho, M. I., Velázquez, J. D., \& Lazarevich, I. (2013). The relationship between sarcopenia, undernutrition, physical mobility and basic activities of daily living in a group of elderly women of Mexico City. Nutricion Hospitalaria, 28(2), 514-521. doi:10.3305/nh.2013.28.2.618

Bann, D., Hire, D., Manini, T., Cooper, R., Botoseneanu, A., McDermott, M. M.,... Gill, T. (2015). Light intensity physical activity and sedentary behavior in relation to body mass index and grip strength in older adults: Cross sectional findings from the Lifestyle Interventions and Independence for Elders (LIFE) Study. PLoS One, 10(2), 1-13. Recuperado de: http://journals.plos.org/ plosone/article?id=10.1371/journal.pone.0116058

Benedetti, T. R. B., Schwingel, A., \& Torres, T. L. (2011). Physical activity acting as a resource for social support among older adults in Brazil. Journal of Human Sport and Exercise, 6(2), 452-461. doi:10.4100/jhse.2011.62.26

Berger, M. J., \& Doherty, T. J. (2010). Sarcopenia: Prevalence, mechanisms, and functional consequences. Interdisciplinary Topics in Gerontology, 37, 94-114. doi: 10.1159/000319997

Bohórquez Gómez-Millán, M.R., Lorenzo Fernández, M. y García González, A.J. (2014). Actividad física como promotor del autoconcepto y la independencia personal en personas mayores. Revista Iberoamericana de Psicología del Ejercicio y el Deporte, 9(2), 533-546. Recuperado de: http:/hdl.handle.net/11441/59364

Cabrero-García, J., Muñoz-Mendoza, C. L., Cabañero-Martínez, M. J., González-Llopís, L., Ramos-Pichardo, J. D., \& Reig-Ferrer, A. (2012). Valores de referencia de la Short Physical Performance Battery para pacientes de 70 y más años en atención primaria de salud. Atención Primaria, 44(9), 540-548.

Cantarero, J. C. \& Carranque, G. (2016). Relación entre el pensamiento creativo y el ejercicio físico en personas adultas. Revista Iberoamericana de Psicología del Ejercicio y el Deporte, 11(1), 47-52. Recuperado de: https://riped-online.com/
index.php?joumal=riped\&page=article\&op=view\&path\%5B\%5D=243

Carrillo-Esper, R., Muciño-Bermejo, Peña-Pérez, C., \& Carrillo-Cortés, U. (2011). Fragilidad y sarcopenia. Revista de la Facultad de Medicina de la UNAM, 54(5), 12-21. Recuperado de: http:// www.medigraphic.com/pdfs/facmed/un-2011/un115c.pdf

Chien, M. Y., Kuo H. K., \& Wu, Y. T. (2010). Sarcopenia, cardiopulmonary fitness, and physical disability in communitydwelling elderly people. Physical Therapy, 90, 1277-1287. Recuperadode:https://academic.oup.com/ptj/article-lookup/doi/10.2522/ ptj.20090322

Cruz-Jentoft, A. J., Baeyens, J. P., Bauer, J. M., Boirie, Y., Cederholm, T., Landi, F.,... Zamboni, M. (2010). Sarcopenia: European consensus on definition and diagnosis / Report of the European Working Group on Sarcopenia in Older People. Age Ageing, 39(4), 412-423. doi: 10.1093/ageing/afq034

Dehghan, M. \& Merchant, A. T. (2008). Is bioelectrical impedance accurate for use in large epidemiological studies?. Open Nutritional Journal, 7(26), 1-7.

Dhalwani, N. N., O’Donovan, G., Zaccardi, F., Hamer, M., Yates, T., Davies, M., \& Khunti, K. (2016). Long term trends of multimorbidity and association with physical activity in older English population. International Journal of Behavioral Nutritona and Physical Activity, 13(8), 1-9. Recuperado de: https:// ijbnpa.biomedcentral.com/articles/10.1186/s12966-016-0330-9

Dodds, R., Kuh, D., Aihie Sayer, A., \& Cooper, R. (2013). Physical activity levels across adult life and grip strength in early old age: updating findings from a British birth cohort. Age \& Ageing, 42(6), 794-798. Recuperado de: https://www.ncbi.nlm.nih.gov/pmc/ articles/PMC3809720/

Enríquez-Reyna, M. C., Cruz-Quevedo, J. E., Celestino-Soto, M. I., Garza-Elizondo, M. E., \& Salazar-González, B. C. (2013). Función ejecutiva, velocidad de marcha y tarea dual en adultos mayores mexicanos. Revista Iberoamericana de Psicología del Ejercicioyel Deporte, 8(2), 345-357. Recuperado de: http://www.webs.ulpgc.es/ riped/docs/20130206.pdf

González-Célis, A. L. (2010). Calidad de vida en el adulto mayor. En Secretaría de Salud, Instituto de Geriatría (Ed). Envejecimiento humano Una visión transdisciplinaria (1a ed.). México: Gobierno de la República.

Goodpaster, B. H., Park, S. W., Harris, T. B., Kritchevsky, S. B., Nevitt, M., Schwartz, A. V.,... Newman, A. B. (2006). The loss of skeletal muscle strength, mass, and quality in older adults: The health, aging and body composition study. The Journals of Gerontology. Series A, Biological Sciences Medicine Sciences, 61(10), 1059-64.

Granic, A., Davies, K., Jagger, C., Kirkwood, T. L., Syddall, H. E., \& Sayer, A.A. (2016). Grip Strength Decline and Its Determinants in the Very Old: Longitudinal Findings from the Newcastle 85+ Study. Plos ONE, 11(9), 1-14. doi:10.1371/journal.pone.0163183

Guillén, F. \& Angulo, J. (2016). Análisis de rasgos de personalidad positiva y bienestar psicológico en personas mayores practicantes de ejercicio físico vs no practicantes. Revista Iberoamericana de Psicología del Ejercicio y el Deporte, 11(1), 113-122. Recuperado de: http://www.redalyc.org/articulo.oa?id=311143051013

Guralnik, J. M., Simonsick, E. M., Ferrucci, L., Glynn, R. J., Berkman, L. F., Blazer, D. G.,... Wallace, R. B. (1994). A short physical performance battery assessing lower extremity function: association with self-reported disability and prediction of mortality and nursing home admission. Journal of Gerontology, 49(2), 85-94.

Hayashida I, Tanimoto Y, Takahashi Y, Kusabiraki T, Tamaki J (2014). Correlation between muscle strength and muscle mass, and Their Association with walking speed, in community-dwelling elderly Japanese Individuals. PLoS One, 9(11), e111810. doi:10.1371/ journal.pone. 0111810

Instituto Nacional de Estadística y Geografía. (2010). Población. Esperanza de vida. Recuperado el 15 de octubre del 2013 de http:// cuentame.inegi.org.mx/poblacion/esperanza.aspx?tema=P 
Instituto Nacional de Salud Pública. (2012). Encuesta Nacional de Salud y Nutrición. Resultados por entidad federativa. Recuperado el 5 de junio de 2018 de:

https://ensanut.insp.mx/informes/NuevoLeon-OCT.pdf

Klee, M. H., Alberici, C., Schûler, C., \& Gonzalez, M. C. (2015). Nutritional status, muscle mass and strength of elderly in Southern Brazil. Nutricion Hospitalaria, 31(1), 363-370. doi: 10.3305/ nh.2015.31.1.7264.

Kohl, H. W., Craig, C. L., Lambert, E. V., Inoue, S., Alkandari, J. R., Leetongin, G., \& Kahlmeier, S. (2012). The pandemic of physical inactivity/: global action for public health. The Lancet, 380(9838), 294-305. doi:10.1016/S0140-6736(12)60898-8

Lauretani, F., Russo, C. R., Bandinelli, S., Bartali, B., Cavazzini, C., Di Iorio, A.,... Ferrucci, L. (2003). Age-associated changes in skeletal muscles and their effect on mobility: an operational diagnosis of sarcopenia. Journal of Applied Physiology, 95(5), 1851-60. Recuperado de: http://jap.physiology.org/content/95/5/1851

Leyton, M., Luis-del-Campo, V., Morenas, J., \& Roldán, A. (2012). Assessment of the level of muscular strength and volume in physically active English adults. Journal of Human Sport and Exercise, 7(1), 296-309. Recuperado de: https://doi.org/10.4100/ jhse.2012.71.08.

Leiva-Caro, J. A., Salazar-González, B. C., Gallegos-Cabriales, E. C., Gómez-Meza, M. V., \& Hunter, K. F. (2015). Connection between competence, usability, environment and risk of falls in elderly adults. Revista Latinoamericana de Enfermagem, 23(6), 11391148. Recuperado de: http://dx.doi.org/10.1590/01041169.0331.2659

Lera-López, F., Garrues, M. A., \& Suárez, M. J. (2017). The correlates of physical activity among the population aged $50-70$ years. Retos, 31, 181-187. Recuperado de: https://recyt.fecyt.es//index.php/retos/article/view/50018

Li-Kuo, L., Wei-Ju, L., Chien-Liang, L., Liang-Yu, C., Ming-Hsien, L., Li-Ning, P., \& Liang-Kung, C. (2013). Age-related skeletal muscle mass loss and physical performance in Taiwan: Implications to diagnostic strategy of sarcopenia in Asia. Geriatrics \& Gerontology International, 13(4), 964-971. doi: 10.1111/ggi.12040

López, R. C., Santos, M. A., Navarro, A. V., Arévalo, J. M., García, F., \& Latorre, P.A. (2016). Determinantes sociodemográficos y nivel de actividad física en la población de la provincial de Jaén mayor de 18 años. Retos, 29, 13-16. Recuperado de: http:// biblioteca.universia.net/html_bura/ficha/params/title/determinantessociodemograficos-nivel-actividad-fisica-poblacion-provincia-jaen18-a\%C3\%B1os/id/65236045.html

Mahoney, F. I. \& Barthel, D. (1965). Functional evaluation: The Barthel Index. Maryland State Medical Journal, 14, 56-61.

Mata-Ordoñez, F., Chulvi-Medrano, I., Heredia-Elvar, J. R., MoralGonzález, S., Marcos-Becerro, J. F., \& Da Silva-Grigogolleto, M. E. (2013). Sarcopenia and resistance training: actual evidence. Journal of Sport and Health Research, 5(1), 7-24. Recuperado de: https:// w w w. re s e a r c h g a t e.net/public a t i o n / 236953592_Sarcopenia_and_resistance_training_actual_evidence

Medina, C., Janssen, I., Campos, I., \& Barquera, S. (2013). Physical inactivity prevalence and trends among mexican adults: results from the National Health and Nutrition Survey (ENSANUT) 2006 and 2012. BMC Public Health, 13(1063), 1-10. Recuperado de: https://bmcpublichealth.biomedcentral.com/articles/10.1186/14712458-13-1063

Montes de Oca, V. (2011). Viudez, soledad y sexualidad en la vejez: mecanismos de afrontamiento y superación. Revista Temática Kairos Gerontología, 14(5), 73-107. Recuperado de: http:// seminarioenvejecimiento.unam.mx/Publicaciones/articulos/ viudez_soledad_sex.pdf

Ortlieb, S., Gorzelniak, L., Nowak, D., Strobl, R., Gril, E., Thorand, B.,... Sculz, H. (2014). Associations between multiple accelerometry-assessed physical activity parameters and selected health outcomes in elderly people - results from the KORA-Age study. PLOS ONE, 9(11), 1-8. Recuperado de: http:// journals.plos.org/plosone/article?id=10.1371/journal.pone.0111206

Patil, R., Uusi-Rasi, K., Pasanen, M., Kannus, P., Karinkanta, S., \& Sievänen, H. (2013). Sarcopenia and osteopenia among 70-80year-old home-dwelling Finnish women: Prevalence and association with functional performance. Osteoporosis International, 24(3), 787-96. doi: 10.1007/s00198-012-2046-2.

Pfeiffer E. (1975). A short portable mental status questionnaire for the assessment of organic brain deficit in elderly patients. Journal of the American Geriatrics Society, 23(10), 433-4. Recuperado de: http://onlinelibrary.wiley.com/doi/10.1111/j.1532$\begin{array}{llllllllllllllllllll}5 & 4 & 1 & 5 & . & 1 & 9 & 7 & 5 & . & \mathrm{t} & \mathrm{b} & 0 & 0 & 9 & 2 & 7 & & \mathrm{x} & /\end{array}$ abstract;jsessionid=0B7F8E12994CF2AF64973AE38A02080B.f03t01

Secretaría de Salud/Instituto Nacional de Salud Pública (2016). Encuesta Nacional de Salud y Nutrición de Medio Camino 2016. Informe final de resultados. Pg. 72. Recuperado el 5 de junio de 2018 de http://promocion.salud.gob.mx/dgps/descargas1/doctos_2016/ ensanut_mc_2016-310oct.pdf

Salvo, D., Torres, C., Villa, U., Rivera, J., Sarmiento, O., Reis, R., \& Pratt, M. (2015). Accelerometer-based physical activity levels among mexican adults and their relation with sociodemographic characteristics and BMI: a cross-sectional study. International Journal of Behavioral Nutritional and Physical Activity, 12(79), 111. Recuperado de: https://ijbnpa.biomedcentral.com/articles/ 10.1186/s12966-015-0243-z

Souza, A. R., Fillenbaum, G. G, \& Blay, S. L. (2015). Prevalence and correlates of physical inactivity among older adults in Rio Grande, do Sul, Brasil. Plos ONE, 10(2), 1-14. Recuperado de: https:// doi.org/10.1371/journal.pone.0117060

Tuero, C. E. \& González-Boto, R. (2018). Factores psicosociales de los usuarios de instalaciones acuáticas: diferencias en función de la edad y el género. Revista Iberoamericana de Psicología del Ejercicio y el Deporte, 13(1), 137-144. Recuperado de: https://riped-

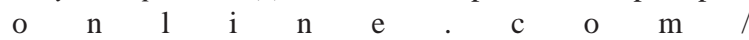
index.php?joumal=riped\&page=article\&op=view\&path\%5B\%5D=515

Washburn, R. A., Smith, K. W., Jette, A. M., \& Janney, C. A. (1993). The Physical Activity Scale for the Elderly (PASE): development and evaluation. Journal of Clinical Epidemiology, 46(2), 153-62.

Zhao, X., Wang, Z., Zhang, J., Hua, J., He, W., \& Zhu, S. (2013) Estimation of total body skeletal muscle mass in Chinese adults: prediction model by Dual-Energy X Ray Absorptiometry. PLoS One, 8(1), e53561. doi:10.1371/journal.pone.0053561

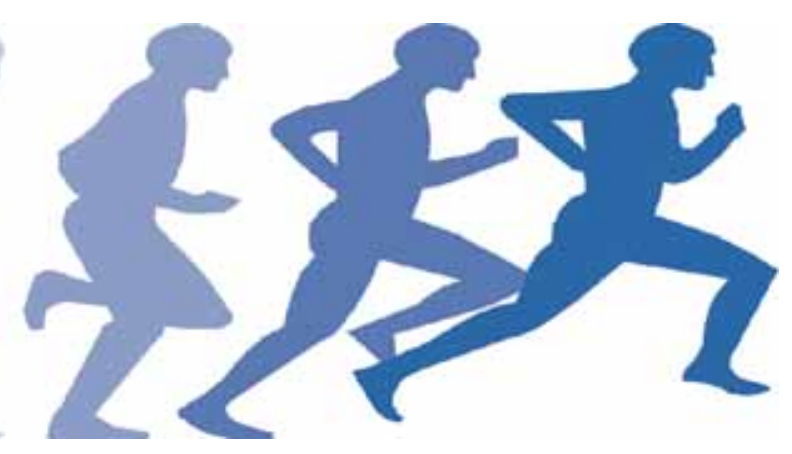

